\title{
From Molecules to Mammals: Fluorescent Proteins for Live Cell and Intravital Imaging
}

*O.M. Subach, *G.S. Filonov, *K.D. Piatkevich, *F.V. Subach, *D.M. Shcherbakova, and *V.V. Verkhusha

* Departments of Anatomy and Structural Biology and Gruss-Lipper Biophotonics Center, Albert Einstein College of Medicine, 1300 Morris Park Avenue, Bronx, NY 10461

The ability of non-invasive monitoring of deep-tissue developmental, metabolic, and pathogenic processes will advance modern biology. Imaging of live mammals using fluorescent probes is more feasible within a "near-infrared optical window". Recently we developed two types of fluorescent probes with emission spectra in the window.

We have engineered a monomeric PSmOrange protein that is initially orange (excitation/emission maxima at 548/565 nm) but becomes far-red (excitation/emission maxima at 636/662 nm) after irradiation with non-cytotoxic blue-green light. Compared to orange proteins, PSmOrange has greater brightness, faster maturation, higher photoconversion contrast, and better photostability. Red-shifted spectra of both PSmOrange forms enable its simultaneous use with cyan-to-green photoswitchable proteins to study dynamics of four intracellular populations. That is impossible with common green-to-red proteins photoswitchable with phototoxic violet light. Compared to red proteins, the photoconverted PSmOrange has the most far-red excitation among GFP-like proteins, provides diffraction-limited and super-resolution PALM images in the far-red shifted range, has optimal excitation with common red lasers, and exhibits higher brightness in mouse. PSmOrange photoconversion occurs via a photo-oxidation process causing a cleavage of the polypeptide backbone. Far-red fluorescence of photoconverted PSmOrange results from a novel chromophore containing $\mathrm{N}$-acylimine with a coplanar carbon-oxygen double bond.

We have developed a dimeric phytochrome-based infra-red fluorescent protein (iRFP) with the excitation/emission maxima at $690 / 713 \mathrm{~nm}$. Bright fluorescence in a living mouse proved iRFP to be a superior probe for non-invasive imaging of internal tissues, while high intracellular stability, low cytotoxicity and lack of the requirement to add external biliverdin-chromophore to fluoresce in cells makes iRFP easy to use as much as conventional GFP-like proteins. Compared to other phytochrome-derived probes, iRFP has better in vitro characteristics and outperforms it in cells and in vivo, having remarkably greater effective brightness and photostability. Compared to the far-red GFP-like proteins, iRFP has substantially higher signal to background ratio in a mouse model owing to its infra-red shifted spectra.

[1] This work was supported by grant GM073913 from the National Institutes of Health to V.V.Verkhusha. 